\title{
Aerial Manipulation: A Literature Review
}

\author{
Fabio Ruggiero, Vincenzo Lippiello, Anibal Ollero
}

\begin{abstract}
Aerial manipulation aims at combining the versatility and the agility of some aerial platforms with the manipulation capabilities of robotic arms. This letter tries to collect the results reached by the research community so far within the field of aerial manipulation, especially from the technological and control point of view. A brief literature review of general aerial robotics and space manipulation is carried out as well.
\end{abstract}

\section{INTRODUCTION}

$\mathbf{T}$ HE INTEREST towards unmanned aerial vehicles (UAVs) is daily growing during the last decade, not only within research communities but also within industrial companies and among public opinion. One may think about the idea that one of the most prominent electronic commerce websites around the world is planning to deliver packages to customers' hands in thirty minutes or less using hexacopters [1]. In 2016, White House has allocated $\$ 35$ million in research funding by the National Science Foundation (NSF) to accelerate the development of design and control of UAVs, especially for civilian uses like monitoring and inspection of physical infrastructures, smart response to disasters, agricultural and meteorological domains [2]. Therefore, applications in which UAVs are employed are continually increasing. As a matter of fact, UAVs are operated to study seismic hazards and areas hit by earthquakes [3]; UAVs are also used by archaeologists to digitally reconstruct the medieval part of Cerreto Sannita, a small town in southern Italy [4]; some companies started to think about a sort of personal UAV equipped with a camera to record self-movies [5], particularly indicated for sportsman.

All the above data and examples lead to the result that aerial robotics is indeed in its golden period. Nevertheless, UAVs have to migrate from passive tasks like inspection, surveillance, monitoring, remote sensing and so on, into active tasks like grasping and manipulation. This perfectly fits what established by the European Robotics Strategic Research Agenda (eSRA), presented in Brussels in 2009 and updated in 2014 [6]. According to the eSRA, aerial and space robots are intended to be employed as robotic workers and co-workers, logistic robots, and robots for exploration and inspection. This means that UAVs have to be endowed with manipulation skills

Manuscript received: September, 10, 2017; Revised December, 11, 2017; Accepted February, 8, 2018.

This paper was recommended for publication by Jonathan Roberts upon evaluation of the Associate Editor and Reviewers' comments. The research leading to these results has been supported by the AEROARMS H2020ICT-2014-1-644271 and HYFLIERS H2020-ICT-2017-1-779411 projects. The authors are solely responsible for the content of this manuscript.

F. Ruggiero and V. Lippiello are with CREATE Consortium and with Department of Electrical Engineering and Information Technology, University of Naples Federico II, via Claudio 21, 80125, Naples, Italy. Anibal Ollero is with the GRVC Robotics Group of the University of Seville, Camino de los Descubrimientos s/n, 41092, Seville, Spain.

First author's email: fabio.ruggiero@unina.it

Digital Object Identifier (DOI): see top of this page.
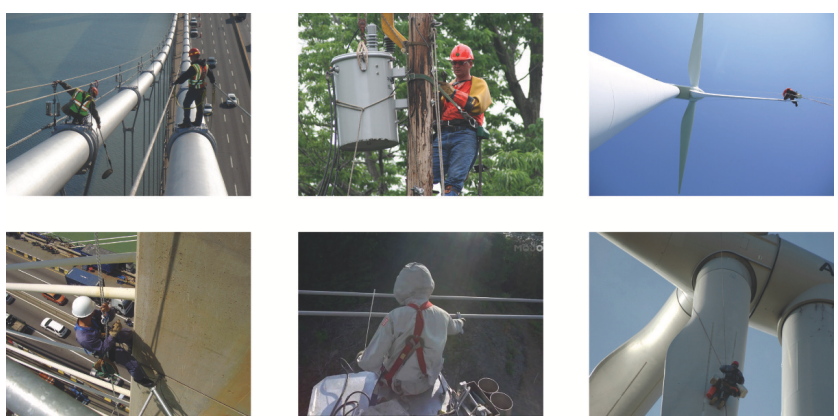

Fig. 1. Application scenarios in which aerial manipulation might be helpful Left column: bridge inspection; central column: high-voltage electric lines inspection and fixing up; right column: rotor blade repairing.

to perform the desired tasks. Hence, from a general point of view, for aerial manipulation it is intended the grasping, transporting, positioning, assembly and disassembly of mechanical parts, measurements instruments and any objects, performed with UAVs. The introduction of aerial manipulation in the scenarios described by the eSRA can be helpful especially in those industrial and service applications that are considered very dangerous for a human operator. For instance, the tasks illustrated in Figure 1 are not only very unsafe, but they are also costly since the performance of professional climbers and specialists might be required. An aerial vehicle able to carry out simple manipulation jobs could indeed assist the human in these activities, or at least in the most hazardous and critical situations.

UAVs should be equipped first of all of the proper tools to accomplish manipulation tasks in the air. The two most adopted solutions are either to mount a gripper or a multifingered hand directly on the aerial vehicle, e.g., a flying hand $(\mathrm{FH})$ or to equip the UAV with one or more robotic arms, e.g., an unmanned aerial manipulator (UAM). In the former case, the object can be grasped and locally manipulated during the flight. However, the single gripper is not enough to obtain a complete evolution from passive to active tasks of the UAVs: mechanical structures mounted on the UAVs are then essential to perform more complex actions. Mobile ground platforms [9], underwater [10], and space robots [11] can be taken as examples of this scenario. Therefore, a UAM could be an efficient solution providing an aerial vehicle with the capability of performing dexterous manipulation tasks.

To the best of the authors' knowledge, the present letter is the first survey about the aerial manipulation topic. Several surveys and textbooks have already been published for the sole UAVs. A brief literature review about the UAVs is any way carried out in the next section because the UAV is the main component of both an FH and a UAM. The solutions employed 

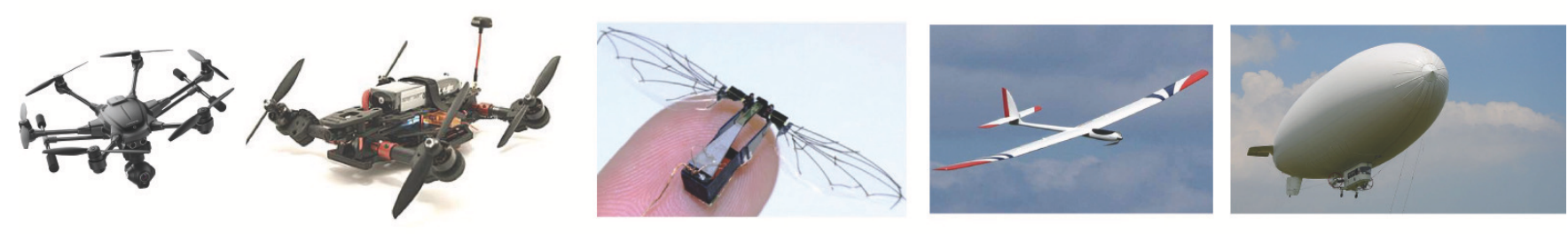

Fig. 2. Examples among the five categories of UAVs described in the text. From left to right: a rotary wings UAV, in particular a hexacopter from YUNEEC [7]; a convertible UAV, in particular a quadrotor with tilting rotors from TILT Racing Drone [8]; a bio-inspired UAV, in particular a robotic flying insect; a fixed-wing $\mathrm{UAV}$, in particular an electric glider; a lighter-than-air UAV, in particular a blimp.

so far to deal with the problems arisen by using these last devices will be instead listed in Section III and Section IV, respectively. A small review about manipulation activities in the space is reported within Section V before the concluding discussion.

\section{BRIEF LITERATURE REVIEW ABOUT CONTROL OF UAVS}

Due to the considerable bulk of applications for aerial vehicles, it is quite difficult to perform an accurate taxonomy of UAVs since there exist many devices in the market with different dimensions, mechanical configuration, actuators and so on. Following what presented in [12], classifying the UAVs from their high manoeuvrability and low endurance toward their low manoeuvrability and high endurance yields: i) rotary wings UAVs (RW-UAVs), like multirotors (e.g., quadcopters, hexacopters), small-scale helicopter-based UAVs (HUAVs), coaxial helicopters and ducted fan UAVs; ii) convertibles UAVs, characterized by interchangeable designs, like tilting rotors or cruise-flight-enable ducted fans UAVs, tail sitters UAVs and so on; iii) bio-inspired UAVs, taking inspiration from the flight of the insects and are mainly concerning flapping wing devices; iv) fixed-wing UAVs, like acrobatic flyers, Delta-wings and electric gliders; v) lighter-than-air UAVs (LtA-UAVs) like autonomous blimps. An example for each of the five categories mentioned above is drawn in Fig. 2. Among the above-listed devices, the vertical take-off and landing (VToL) UAVs do not need a runway to both detach and land from/to the ground. Compared to standard aircrafts, multirotors UAVs are low-cost devices and easily maneuverable. They can perform hovering in a precise way, but the endurance is not their best peculiarity. Rotary wings VToL UAVs are of interest within this literature review. Two main issues have been tackled by the research community when dealing with them.

The first is that conventional VToL devices, such as multirotors UAVs with parallel axis, are underactuated and this establishes several problems in stabilizing the vehicle and tracking the desired trajectories. The most widely used controller takes into account a hierarchical architecture [13], [14] highlighting a time-scale separation between the linear (slow time-scale) and angular (fast time-scale) dynamics. Moreover, it is possible to show that the position and the yaw angle of the VToL UAV are flat outputs [15], [16]. Hence, it is possible to find a set of inputs to track any trajectory in the Cartesian space with a desired heading angle of the UAV. This solves the underactuation problem since tracking of the flat outputs (slow time-scale part of the system) generates the references for the low-level attitude controller (fast time-scale part of the system). Other worthy approaches rely upon backstepping [17], impedance [18] and optical flow [19] techniques.

The second issue is that the aerodynamic model of UAVs is very complicated and several assumptions are made during its derivation. This leads to robust control designs that are worthy of interest within UAMs (see Section IV-B). Most of them implement an integral action to resist against external disturbances and cope with unknown and time-varying parameters (e.g., the battery level). Recently, adaptive controls have been employed to counteract such disturbances [20], [21], [22], [23], [24], [25]. A nonlinear force observer is introduced in [26] to estimate disturbances applied to a quadrotor. A sliding mode observer is instead employed in [27] to impose more robustness on the closed-loop system. An attitude estimator coping with magnetic disturbances and the bias of the gyrometers is proposed in [28]. Since passivity-based controllers do not rely on the exact compensation of the considered model, they are expected to be more robust to parameters uncertainties. Port-Hamiltonian methods are developed in [26], [29], [30], a passive backstepping in [31], and passivitybased attitude controls in [32], [33], in particular without angular velocity measurement in [34], [35]. A momentumbased compensation of external wrench (force plus moment) is introduced in [36], together with an impedance controller, to reduce unmodelled effects and external disturbances further. A hybrid estimation is instead proposed in [37], which does not require the translational drift velocity of the UAV as an input parameter.

A broader literature review about this topic can be found in seminal works like the review on control and perception techniques for aerial robotics in [38], the survey on smallscale UAVs in [39] and VToL UAVs in [40], or particularly quadrotors in [41]. Many textbooks have also been published on the topic, like [13], [42], [43], and a handbook [44].

\section{FLYING HANDS}

Within the class of FHs, it is possible to consider those platforms in which the carried object cannot be moved independently from the UAV. Therefore, two cases can be in principle considered: i) the object, or a rigid tool like a gripper, 


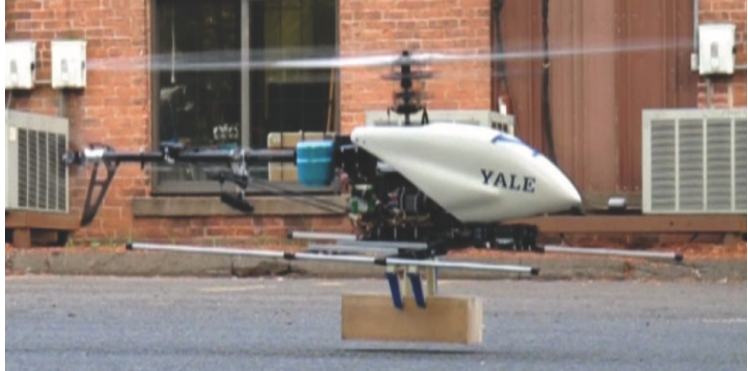

Fig. 3. Example of a flying hand, in particular the Yale Aerial Manipulator capturing a block in hover (picture taken from [45]).

is directly attached to the UAV; ii) the object is linked to the aerial vehicle through some cables or tether mechanisms. An example of $\mathrm{FH}$ is represented in Fig. 3.

Gripper directly attached to the UAV: The first question to address once a gripper must be attached to the UAV is its mechanical design. Usually, UAV's payload is limited, and the battery consumption increases once the total weight of the FH approaches the limit. It is easily understood that the bigger the carried payload, the bigger should be the capacity of the single employed UAV. Since an FH can only grasp an object and locally manipulate it without too much dexterity, it is not convenient to complicate the mechanical design of the gripper. Light-weight, low-complexity grippers are conceived for quadrotors [46] and helicopters [47], even investigating low-cost solutions [48]. Besides, these designed grippers for FHs can be endowed with compliance concerning the external environment [45], [48], [49] or not [46].

A further issue arising during the mechanical assembly of an FH is where to place the gripper on the UAV. Regarding the quadrotor, the rigid tool should be positioned above and not below the aerial vehicle [50]. However, although this solution is to be preferred from a theoretical point of view for the internal stabilization of the $\mathrm{FH}$, it raises some problems in practical applications where it would be difficult for an $\mathrm{FH}$ to grasp an object by approaching it from below.

During flight operations, it is intuitive that the coupling between the grasped object and the aerial platform arises several problems, like the destabilization of the UAV, also due to the underactuation of the aerial vehicle and its intrinsically unstable dynamics. Therefore, challenges encountered during the take-off and the free-flight phases are addressed in [45], [51] when a helicopter, or a quadrotor, transports a load through a fixed gripper: stability bounds are derived not to destabilize the employed flight controller. Besides, a hybrid force/motion control framework is designed in [52] for a rigid tool attached to the UAV.

Usually, the reviewed literature tends to include within the class of FHs many devices having a rigid tool interacting with the environment [53], [54]. Direct applications can be easily seen in tasks like pushing [55], door opening [56] and aerial writing [57]. The use of multiple aerial-ground manipulator systems (MAGMaS) where one or more FHs grab an object together with ground manipulators [58] is of interest as well.
Cables or tether mechanisms: Those devices where the object is transported through cables by the aerial platforms can be considered as FHs as well. The problem of lifting a load from the ground through a cable connected to the UAV is addressed in [59], [60]. An adaptive control to render the flight agiler is exploited in [21] along with an optimal trajectory generation. A fast trajectory optimization is instead tackled in [61]. Analogous problems are faced by aerial robots tethered by cables and/or bars to a (moving) platform [62], [63], [64], [65]. A textbook collecting the latest results in planning trajectory and control for UAVs with suspended payloads is in press [66]. The case of multiple cooperative UAVs transporting an object has also been widely investigated [67], [68], [69], [70].

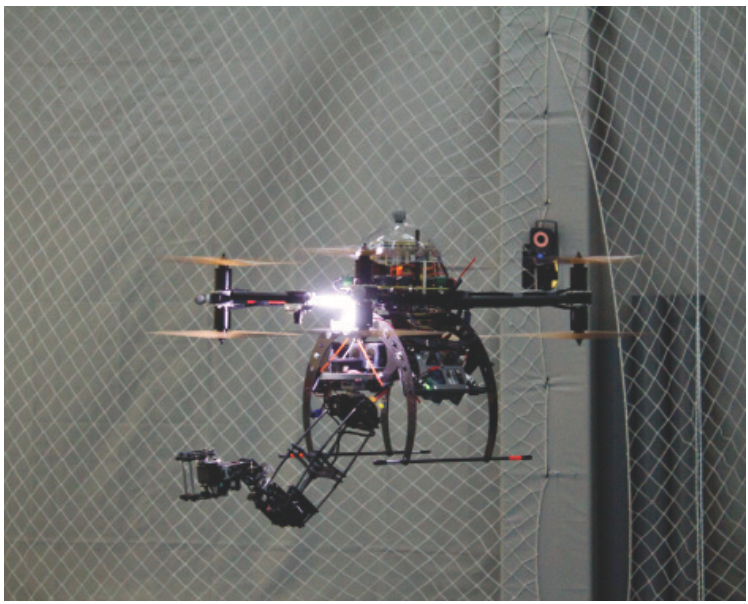

Fig. 4. Unmanned aerial manipulator composed by a multirotor, an eight rotor aircraft in coaxial configuration, equipped by a 6 -DoF servo-driven manipulator, developed at CATEC within the ARCAS project [71]. More details about this UAM can be found in [72], [73].

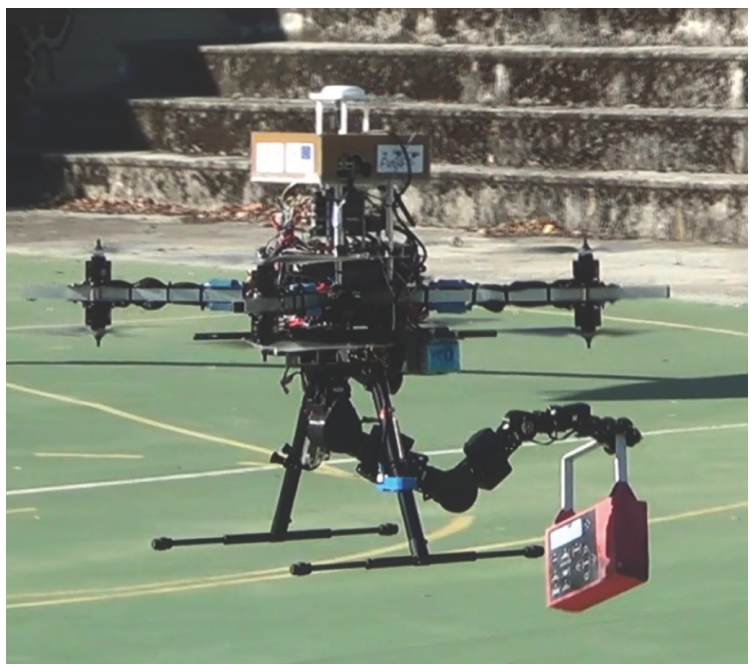

Fig. 5. Unmanned aerial manipulator composed by a multirotor, an eight rotor aircraft in coaxial configuration, equipped by a 7 -DoF servo-driven manipulator, developed at University of Seville within the ARCAS project [71]. More details about this UAM can be found in [74]. 


\section{UNMANNED AERIAL MANIPULATORS}

From the previous section, it is possible to infer the concept that through an FH it is not possible to do more than pick-andplace operations. As revised in this section, the opportunities provided by UAMs may be fruitful to increase dexterity within aerial manipulation tasks.

Four elements mainly constitute a UAM: i) the UAV floating base; ii) the robotic arm; iii) a gripper or a multi-fingered hand attached at the end-effector of the arm; iv) other sensors like cameras, laser scanners, and so on. Figure 4 and Figure 5 illustrate the UAM concept.

Likewise the analysis carried out in the previous section, the design of the mechanical arm is a crucial aspect also for the UAMs. Moreover, the more dexterity can be added to the aerial platform, the more solutions can be thought to design an efficient UAM. Therefore, it is desirable categorizing a UAM based on the different technological solutions that can be found within the literature addressing the problem of mounting a robot manipulator on a UAV.

1) A first distinction can be performed on the number of DoFs of the employed arm: 1 DoF [75], 2 DoFs [76] or more [72], [74].

2) A second distinction can be made based on the mechanics of the joints of the robot arm. Most of the employed joints are rotational: few examples of UAMs showing prismatic joints can be found [77], [78], also displaying compliant behaviors [79].

3) A further distinction can be made in the way how the motors are controlled: directly in position, velocity, acceleration, or torque-controlled motors [80].

4) Finally, a distinction can be made on the basis of the resulting configuration: for instance, a Delta-like structure is employed in [81], a parallel manipulator is considered in [82], a hyper-redundant 9 DoFs robot arm is designed in [83], while a redundant 7 DoFs fully actuated anthropomorphic robot arm like the KUKA LWR is employed in [84].

During flight operations, the presence of a carried object creates coupling effects in the dynamic model of the system. A mounted robot arm provides even more issues since its dynamics depends on the actual configuration state of the whole system. Basically, there are two approaches to address planning and control problems for a UAM. The former is a centralized approach, in which the UAV and the robotic arm are considered as a unique entity, and thus the planning and the controller are designed from the complete kinematic and dynamic models. The latter approach considers the UAV and the robotic arm as two separate independent systems. The effects of the arm on the aerial vehicle are then considered as external disturbances and vice versa. This approach might be useful in case the dynamics of the arm is not enough to compensate the UAV position error and/or in case the arm does not allow torque control. The two next subsections follow the above-sketched distinction in revising the related scientific literature.

Notice that the same distinction can be made to control UAMs with multiple arms attached to the aerial vehicle. This

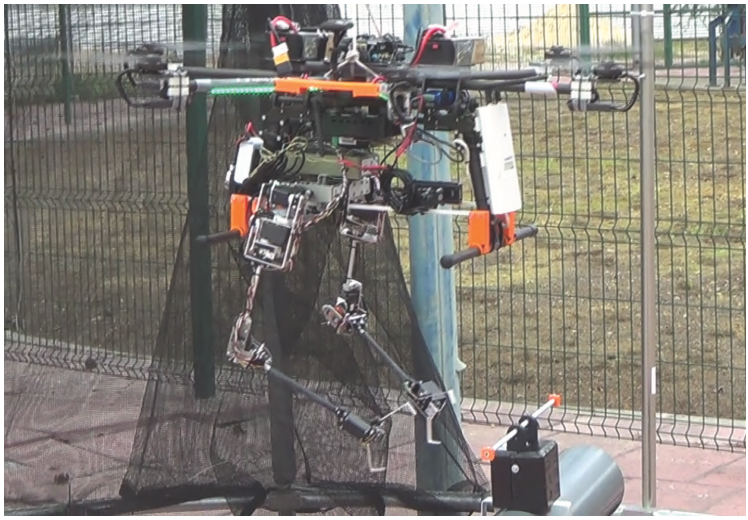

Fig. 6. A UAM with a dual-arm system, developed and designed by the University of Seville within the AEROARMS project [85]. Details of the preliminary version can be found in [86], while the most advanced version including visual servoing is described in [87].

configuration may be useful to bestow the system with higher manipulation capabilities which could be required in several inspection and maintenance activities. The use of multiple arms on a UAV may extend the range of possible application thanks to the increased ability of grab and dock on prohibitive locations, while still perform dexterous accurate manipulation with another arm. A further design is the possibility to have a robot arm plus one or more articulated clamping devices on the same aerial platform. As for the UAM with a single arm, the design of a lightweight and human-size system is essential [86]. The case of multiple arms in which all the respective first joints are attached at the UAV's center of mass is investigated in [88], where it is demonstrated that such a peculiar design is a differentially flat system. Regarding carried out applications with a dual arm UAM is worth mentioning the aerial valve-turning task developed in [89], [90]. Recently, the PRODRONE company has unveiled an UAM with a dual robot arm large-format drone [91]. The first developed autonomous prototype for research centers is instead represented in Fig. 6.

\section{A. Centralized approach}

Since the aerial platform and the robotic arm are seen as a paired entity, the first step is the derivation of its dynamic model. Typically, two approaches can be followed: the standard Euler-Lagrangian formalism, in which it is possible to have a symbolic matrix form of the whole dynamic model [92], [93], and the Newton-Euler recursive formulation which has more simplifications from a coding point of view [94], [95].

Regardless the method employed to derive the dynamic model, several model-based approaches have been developed so far to cope with the issues drafted above. A simple fullstate feedback LQR is designed near the equilibrium point of the whole dynamic system in [75]. An adaptive sliding mode controller is instead introduced in [76]. A backstepping-based controller for the UAM that uses the coupled full dynamic model is addressed in [74], while an admittance controller for the manipulator arm is designed. An output feedback linearization and stable zero dynamics are exploited in [78]. 
A combination of gain-scheduling and Lyapunov-based model reference adaptive control (MRAC) is addressed in [96]. A hierarchical controller, employing a closed-loop inverse kinematic algorithm (CLIK) within the first layers, is designed in [97]. An impedance filter based on the wrench measures provided by a sensor mounted on the arm's wrist, an inverse kinematic module and a motion controller are combined to reduce the interaction forces of the end-effector with the environment [98]. Further theoretical work has revealed an underlying structure of the centralized dynamics helping the control design [75]. Depending on the employed UAM, it is possible that the mechanical system is redundant for the given task. Therefore, a Cartesian impedance control with redundancy resolution is described in [93]. Notice that even if the UAM is redundant for the task, the underactuation problem regarding the actuation of the UAV persists.

Concerning the applications where UAMs have been employed following such centralized approach, a simulated hole insertion has been investigated in [99], while an aerial valveturning task is instead inspected in [100].

\section{B. Decentralized approach}

In case the aerial platform and the attached robot arm are seen as two independent entities, the mechatronic and control problems are addressed separately. On the one hand, the control of the sole robot manipulator is well-established in the literature [101], and it is out of the scope of this letter. On the other hand, the control of the single UAV has already been examined briefly in Section II.

Since the two main components of the UAM are controlled independently, robust control of both the UAV and the robot arm is sought because the movements of the robot arm are seen as a perturbation for the aerial platform and vice versa. Suitable disturbance observers and robust controller are thus adequately designed in a decentralized approach for UAMs. The literature review reveals a multilayer architecture to control multirotor UAVs equipped with a servo robot arm [73], where the momentum-based observer presented in [36], [102] is employed to compensate neglected aerodynamic effects and the arm dynamics. A variable parameter integral backstepping controller improves the results obtained by a simple PIDbased controller for the UAV [103]. A UAM composed of a helicopter and a fully actuated redundant robot arm (a Kuka LWR with 7 DoFs) does not show coupling effects when the center of gravity of the arm is moved in the lateral plane of the helicopter: forcing the movement of the arm in that direction, thanks to its intrinsic redundancy, exhibits a coupling between the UAV and the manipulator only at a kinematic level [84].

\section{BRIEF LITERATURE REVIEW ABOUT SPACE MANIPULATION}

Space manipulation shares several open issues with aerial manipulation like mobility, teleoperation, and autonomy, additionally coping with extreme environments (microgravity, extreme temperatures, high vacuum, fine dust, high pressure, corrosive atmospheres, radiations, and so on). Hence, it is worth briefly revising the related literature.
Historical developments for space robotics systems can be found in [11]. Four categories of space manipulators are typically considered: i) free-floating manipulator systems, where the platform fluctuates due do the movements of the moving manipulators, and the kinetic momentum of the system is conserved if no external wrench is applied; ii) macro-mini manipulator systems, composed of a relatively small arm for fine manipulation mounted on a more prominent arm for positioning; iii) flexible-based manipulator systems, that is as a macro-mini manipulator with the macro arm acting as an elastic structure; iv) mobile robots with articulated limbs, like wheeled vehicles or walking robots for exploration of the planetary surface. It is easily recognizable that the first category has affinities with a UAM.

Among various applications within space manipulation, it is worth citing the problem of grasping a target satellite through a free-floating manipulator system. The target satellite is uncontrolled and non-cooperative. Such grasping task may be solved within the context of optimal or nonlinear control [104], [105], [106], with use of the onboard sensor signals, or in that of telepresence control, with a human operator in the loop [107]. By employing a satellite's motion estimation parameters [108], a nonlinear optimization algorithm for optimally solving the task is presented in [109] to overcome the presence of local solutions.

In conclusion, space robotics does not have a structured environment like industrial applications, and it shares several issues of aerial manipulation with UAMs. However, hardware handled by space robots is much more expensive and very delicate. Performing repetitive experiments is not suitable, and it is indeed very complicated for logistic reasons. Recreate on the Earth zero/micro-gravity conditions is also costly and not so doable in every research center. Simulations should be very sophisticated to reduce the testing time on real space floating platforms.

\section{CONCLUSION}

Aerial manipulation can be considered as the natural evolution of mobile robotics, adding manipulation capabilities to the versatility and agility of VToL UAV, as well also to floating space platform and bigger UAV such as helicopters and so on. Without doubts, this will improve the quality of the job of many workers operating in dangerous and hazardous conditions and situations.

However, it is also evident that a lot of work is still on the way. Several deductions can be concluded from the above literature review. In general, energy and safety issues are two main limitations. The lack of high accuracy is also relevant in some applications. A significant challenge of the next decade is doubtlessly related to power consumption and short-lived batteries, but this is out of the scope of this letter. On the other hand, uncertified aerial devices are forcing several countries to introduce proper restriction laws to limit the use of drones in open and crowded spaces for safety reasons. As pointed out by the SPRINT Robotics roadmap [110], such aerial devices must instead be able to work in real-world scenarios, opposed to almost organised laboratory environments, in which the 
weather may be inclement and the equipment must be adequately verified (i.e., it must be explosion proof through ATEX or IECEx certifications).

Therefore, mechatronics for both FHs and UAMs is indeed a crucial aspect. Despite the results pointed out throughout the above literature review, significant challenges are still under development to improve the safety and energetic issues sketched above. Further investigation is needed to strengthen the connection between the mechanical system design and the performance of the aerial manipulator in real applications. Mechanical design and control should thus be conceived together and not as two separate steps. As an example, while it has been discovered that a grasping tool should place above a quadrotor to increase internal stability, a similar study is missing for the UAMs to the best of authors' knowledge. Hybrid mechatronic solutions combining the best part of a single configuration may also be conceived.

The SPRINT roadmap mentioned above also highlights some opportunities for robots, and of course aerial manipulator devices, in inspection and maintenance. Citing some examples with particular reference to the aerial robotic domain, robots can help/replace human operators on remote and offshore sites; robots can operate in hazardous tasks like reaching the highest places of a plant or a building; robots can help in scaffolding that usually is associated with a risk for the human health; aerial devices can increase the total number of inspections of a plant, monitoring the wear of the components. These sought achievements should be reached not only by coping with the issues outlined above but also by improving the environmental performance. However, aerial manipulation activities are prerogative of universities and research centers so far. To counteract this, recently, the European community has financed several projects within latest funding programs in remote aerial inspection (AIRobots, [111]), cooperative robot system assembly and structure construction (ARCAS, [71]), mixed ground and aerial robotic platform to support search and rescue activities (SHERPA, [112]), robotics challenge for plant servicing and inspection using aerial technology (EuRoC, [113]), collaborative aerial robotic workers (Aeroworks, [114]), aerial robotic system integrating multiple arms and advanced manipulation capabilities for inspection and maintenance (AEROARMS, [85]). An innovation from AEROARMS project has recently been the Overall Winner of the European Commission Innovation Radar Prize [115]. These projects tried, or are trying, to reduce the gap for a fast technology migration from academia to industry, but it is still a challenging road.

\section{REFERENCES}

[1] Amazon Prime Air, http://www.amazon.com/b?node=8037720011.

[2] The White House, https://obamawhitehouse.archives.gov/the-pressoffice/2016/08/02/fact-sheet-new-commitments-accelerate-safeintegration-unmanned-aircraft.

[3] Geo Social - Science \& Media, http://www.geo-social.net/?p=829.

[4] Il Mattino, http://www.ilmattino.it/BENEVENTO/benevento-dronecerreto-sannita/notizie/1122700.shtml.

[5] IEEE Spectrum, http://spectrum.ieee.org/aerospace/aviation/flyingselfie-bots-tagalong-video-drones-are-here/.

[6] The Parternship for Robotics in Europe. The Strategic Research Agenda, http://www.eurobotics.net/cms/upload/PPP/SRA2020_SPARC.pdf.
[7] Yuneec, https://www.yuneec.com.

[8] TILT Racing Drone, http://www.tiltdrone.com.

[9] Y. Yamamoto and X. Yun, "Coordinating locomotion and manipulation of a mobile manipulator," IEEE Transactions on Automatic Control, vol. 39, no. 6, pp. 1326-1332, 1994.

[10] G. Antonelli, Underwater Robotics. Motion and Force Control of Vehicle-Manipulator Systems, ser. Springer Tracts in Advanced Robotics. Berlin Heidelberg, D: Springer-Verlag, 2006, vol. 2.

[11] K. Yoshida, B. Wilcox, G. Hirzinger, and R. Lampariello, "Space robotics," in Springer Handbook of Robotics, B. Siciliano and O. Khatib, Eds. Springer International Publishing, 2016, pp. 14231461.

[12] S. Leutenegger, C. Hürzeler, A. Stowers, K. Alexis, M. Achtelik, D. Lentink, P. Oh, and R. Siegwart, "Flying robots," in Springer Handbook of Robotics, B. Siciliano and O. Khatib, Eds. Springer International Publishing, 2016, pp. 623-669.

[13] K. Nonami, F. Kendoul, S. Suzuki, and W. Wang, Autonomous Flying Robots. Unmanned Aerial Vehicles and Micro Aerial Vehicles. Berlin Heidelberg, D: Springer-Verlag, 2010.

[14] R. Mahony and T. Hamel, "Robust trajectory tracking for a scale model autonomous helicopter," International Journal of Robust and Nonlinear Control, vol. 14, no. 12, pp. 1035-1059, 2004.

[15] M. Fliess, J. Levine, P. Martin, and P. Rouchon, "Flatness and defect of nonlinear systems: Introductory theory and examples," International Journal of Control, vol. 61, no. 6, pp. 1327-1361, 1995.

[16] R. Spica, A. Franchi, G. Oriolo, H. Bülthoff, and P. Robuffo Giordano, "Aerial grasping of a moving target with a quadrotor UAV," in 2012 IEEE/RSJ International Conference on Intelligent Robots and Systems, Vilamoura, P, 2012, pp. 4985-4992.

[17] T. Madani and A. Benallegue, "Backstepping control for a quadrotor helicopter," in IEEE/RSJ International Conference on Intelligent Robots and Systems, Beijing, C, 2006, pp. 3255-3260.

[18] M. Fumagalli and R. Carloni, "A modified impedance control for physical interaction of UAVs," in 2013 IEEE/RSJ International Conference on Intelligent Robots and Systems, Tokyo, J, 2013, pp. 1979-1984.

[19] V. Lippiello, G. Loianno, and B. Siciliano, "MAV indoor navigation based on a closed-form solution for absolute scale velocity estimation using optical flow and inertial data," in 50th IEEE Conference on Decision Control and European Control Conference, Orlando, FL, USA, 2011, pp. 3566-3571.

[20] A. Roberts and A. Tayebi, "Adaptive position tracking of VTOL UAVs," IEEE Transactions on Robotics, vol. 27, no. 1, pp. 129-142, 2011.

[21] I. Palunko, P. Cruz, and R. Fierro, "Agile load transportation. Safe and efficient load manipulation with aerial robots," Robotics and Automation Magazine, vol. 19, no. 3, pp. 69-79, 2012.

[22] G. Antonelli, E. Cataldi, P. Robuffo Giordano, S. Chiaverini, and A. Franchi, "Experimental validation of a new adaptive control scheme for quadrotors MAVs," in 2013 IEEE/RSJ International Conference on Intelligent Robots and Systems, Tokyo, J, 2013, pp. 3496-3501.

[23] G. Antonelli, F. Arrichiello, S. Chiaverini, and P. Robuffo Giordano, "Adaptive trajectory tracking for quadrotor MAVs in presence of parameter uncertainties and external disturbances," in Proceedings 2013 IEEE/ASME International Conference on Advanced Intelligent Mechatronic, Wollongong, AU, 2013, pp. 1337-1342.

[24] Z. Dydek, A. Annaswamy, and E. Lavretsky, "Adaptive control of quadrotor UAVs: A design trade study with flight evaluations," IEEE Transactions on Control Systems Technology, vol. 21, no. 4, pp. 14001406, 2013.

[25] D. Cabecinhas, R. Cunha, and C. Silvestre, "A nonlinear quadrotor trajectory tracking controller with disturbance rejection," Control Engineering Practice, vol. 26, pp. 1-10, 2014.

[26] B. Yüksel, C. Secchi, H. Bülthoff, and A. Franchi, "A nonlinear force observer for quadrotors and application to physical interactive tasks," in 2014 IEEE/ASME International Conference on Advanced Intelligent Mechatronics, Besançon, F, 2014, pp. 433-440.

[27] T. Madani and A. Benallegue, "Sliding mode observer and backstepping control for a quadrotor unmanned aerial vehicles," in Proceedings of the 2007 American Control Conference, New York City, NY, USA, 2007, pp. 5887-5892.

[28] M.-D. Hua, G. Ducard, T. Hamel, R. Mahony, and K. Rudin, "Implementation of a nonlinear attitude estimator for aerial robotic vehicles," IEEE Transactions on Control Systems Technology, vol. 22, no. 1, pp. 201-213, 2014.

[29] R. Mahony, S. Stramigioli, and J. Trumpf, "Vision based control of aerial robotic vehicles using the port Hamiltonian framework," in 
50th IEEE Conference on Decision Control and European Control Conference, Orlando, FL, USA, 2011, pp. 3526-3532.

[30] B. Yüksel, C. Secchi, H. Bülthoff, and A. Franchi, "Reshaping the physical properties of a quadrotor through IDA-PBC and its application to aerial physical interaction," in 2014 IEEE International Conference on Robotics and Automation, Honk Kong, C, 2014, pp. 6258-6265.

[31] C. Ha, Z. Zuo, F. Choi, and D. Lee, "Passivity-based adaptive backstepping control of quadrotor-type UAVs," Robotics and Autonomous Systems, vol. 62, no. 9, pp. 1305-1315, 2014.

[32] O. Egeland and J.-M. Godhavn, "Passivity-based adaptive attitude control of a rigid spacecraft," IEEE Transactions on Automatic Control, vol. 39, no. 4, pp. 842-846, 1994.

[33] S. Formentin and M. Lovera, "Flatness-based control of a quadrotor helicopter via feedforward linearization," in 2011 50th IEEE International Conference on Decision and Control and European Control Conference, Orlando, FL, USA, 2011, pp. 6171-6176.

[34] F. Lizarralde and J. T. Wen, "Attitude control without angular velocity measurement: A passivity approach," IEEE Transactions on Automatic Control, vol. 41, no. 3, pp. 468-472, 1996.

[35] P. Tsiotras, "Further passivity results for the attitude control problem," IEEE Transactions on Automatic Control, vol. 43, no. 11, pp. 15971600, 1998.

[36] F. Ruggiero, J. Cacace, H. Sadeghian, and V. Lippiello, "Impedance control of VToL UAVs with a momentum-based external generalized forces estimator," in 2014 IEEE International Conference on Robotics and Automation, Hong Kong, C, 2014, pp. 2093-2099.

[37] T. Tomic and S. Haddadin, "A unified framework for external wrench estimation, interaction control and collision reflexes for flying robots," in 2014 IEEE/RSJ International Conference on Intelligent Robots and Systems, Chicago, IL, USA, 2016, pp. 561-566.

[38] A. Ollero and L. Merino, "Control and perception techniques for aerial robotics," Annual Reviews in Control, vol. 28, no. 2, pp. 167-178, 2004.

[39] G. Cai, J. Dias, and L. Seneviratne, "A survey of small-scale unmanned aerial vehicles: Recent advances and future development trends," Unmanned Systems, vol. 2, no. 2, pp. 175-199, 2014.

[40] M.-D. Hua, T. Hamel, P. Morin, and C. Samson, "Introduction to feedback control of underactuated VTOL vehicles: A review of basic control design ideas and principles," IEEE Control Systems Magazine, pp. $61-75,2013$

[41] S. Gupte, P. Teenu Mohandas, and J. Conrad, "A survey on quadrotor unmanned aerial vehicles," in 2012 IEEE Southeastcon, Orlando, FL, USA, 2012.

[42] R. Austin, Unmanned air systems: UAV design, development and deployment. Wiley, 2010.

[43] K. Valvanis, Advances in unmanned aerial vehicles: State of the art and the road to autonomy, ser. Intelligent Systems, Control and Automation: Science and Engineering. Springer Netherlands, 2007, vol. 33 .

[44] K. Valvanis and G. Vachtsevanos, Handbook of unmanned aerial vehicles. Springer Netherlands, 2015.

[45] P. Pounds, D. Bersak, and A. Dollar, "Grasping from the air: Hovering capture and load stability," in 2011 IEEE International Conference on Robotics and Automation, Shanghai, C, 2011, pp. 2491-2498.

[46] D. Mellinger, Q. Lindsey, M. Shomin, and V. Kumar, "Design, modeling, estimation and control for aerial grasping and manipulation," in 2011 IEEE/RSJ International Conference on Intelligent Robots and Systems, San Francisco, CA, USA, 2011, pp. 2668-2673.

[47] S. Backus, L. Odhner, and A. Dollar, "Design of hands for aerial manipulation: Actuator number and routing for grasping and perching," in 2014 IEEE/RSJ International Conference on Intelligent Robots and Systems, Chicago, IL, USA, 2014, pp. 34-40.

[48] V. Ghadiok, J. Goldin, and W. Ren, "Autonomous indoor aerial gripping using a quadrotor," in 2011 IEEE/RSJ International Conference on Intelligent Robots and Systems, San Francisco, CA, USA, 2011, pp. 4645-4651.

[49] P. Pounds and A. Dollar, "Hovering stability of helicopters with helastic constraints," in 2010 Dynamic Systems and Control Conference, Cambridge, MA, USA, 2010, pp. 781-788.

[50] H. Nguyen, C. Ha, and D. Lee, "Mechanics, control and internal dynamics of quadrotor tool operation," Automatica, vol. 61, pp. 289 301,2015

[51] P. Pounds, D. Bersak, and A. Dollar, "Stability of small-scale UAV helicopters and quadrotors with added payload mass under PID control," Autonomous Robots, vol. 33, no. 1-2, pp. 129-142, 2012.

[52] H. Nguyen and D. Lee, "Hybrid force/motion control and internal dynamics of quadrotors for tool operation," in 2013 IEEE/RSJ Interna- tional Conference on Intelligent Robots and Systems, Tokyo, J, 2013, pp. 3458-3464.

[53] S. Bellens, J. De Schutter, and H. Bruyninckx, "A hybrid pose/wrench control framework for quadrotor helicopters," in 2012 IEEE International Conference on Robotics and Automation, Saint Paul, MN, USA, 2012, pp. 2269-2274.

[54] A. Mersha, S. Stramigioli, and R. Carloni, "Variable impedance control for aerial interaction," in 2014 IEEE/RSJ International Conference on Intelligent Robots and Systems, Chicago, IL, USA, 2014, pp. 34353440.

[55] C. Papachristos, K. Alexis, and A. Tzes, "Efficient force exertion for aerial robotic manipulation: Exploiting the thrust-vectoring authority of the tri-tiltoror UAV," in 2014 IEEE International Conference on Robotics and Automation, Honk Kong, C, 2014, pp. 4500-4505.

[56] H. Tsukagoshi, M. Watanabe, T. Hamada, D. Ashlih, and R. Iizuka, "Aerial manipulator with perching and door-opening capability," in 2015 IEEE International Conference on Robotics and Automation, Seattle, WA, USA, 2015, pp. 4663-4668.

[57] G. Darivianakis, K. Alexis, M. Burri, and R. Siegwart, "Hybrid predictive control for aerial robotic physical interaction towards inspection operations," in 2014 IEEE International Conference on Robotics and Automation, Honk Kong, C, 2014, pp. 53-58.

[58] N. Staub, M. Mohammadi, D. Bicego, D. Prattichizzo, and A. Franchi, "Towards robotic MAGMaS: Multiple aerial-ground manipulator systems," in 2017 IEEE International Conference on Robotics and Automation, Singapore, 2017, pp. 1307-1312.

[59] I. Palunko and R. Fierro, "Autonomous lift of a cable-suspended load by an unmanned aerial robot," in 2014 IEEE Conference on Control Applications, Antibes, F, 2014, pp. 802-807.

[60] P. Cruz and R. Fierro, "Cable-suspended load lifting by a quadrotor UAV: Hybrid model, trajectory generation, and control," Autonomous Robot, vol. 41, pp. 1629-1643, 2017.

[61] P. Foehn, D. Falanga, N. Kuppuswamy, R. Tedrake, and D. Scaramuzza, "Fast trajectory optimization for agile quadrotor maneuvers with a cable-suspended payload," in Robotics: Science and Systems, Cambridge, MA, USA, 2017.

[62] L. Sandino, D. Santamaria, M. Bejar, K. Kondak, A. Viguria, and A. Ollero, "First experimental results on enhancing hovering performance of unmanned helicopters by using a tethered setup," Robotics and Autonomous Systems, vol. 79, pp. 147-155, 2016.

[63] M. Tognon, A. Testa, E. Rossi, and A. Franchi, "Takeoff and landing on slopes via inclined hovering with a tethered aerial robot," in 2016 IEEE/RSJ International Conference on Intelligent Robots and Systems, Daejon, ROK, 2016, pp. 1702-1707.

[64] S. Tognon, M. Dash and A. Franchi, "Observer-based control of position and tension for an aerial robot tethered to a moving platform," IEEE Robotics and Autonomous Letters, vol. 1, no. 2, pp. 732-737, 2016.

[65] M. Tognon and A. Franchi, "Dynamics, control, and estimation for aerial robots tethered by cables and bars," IEEE Transactions on Robotics, vol. 33, no. 4, pp. 834-845, 2017.

[66] I. Palunko, P. Cruz, and R. Fierro, Load transportation using aerial robots, ser. SpringerBriefs in Applied Sciences and Technology. Springer International Publishing, 2018.

[67] M. Bernard, K. Kondak, I. Maza, and A. Ollero, "Autonomous transportation and deployment with aerial robots for search and rescue mission," Journal of Field Robotics, vol. 28, no. 6, pp. 914-931, 2011.

[68] N. Michael, J. Fink, and V. Kumar, "Cooperative manipulation and trasportation with aerial robots," in Autonomous robots. Special issue: Robotics: Science and Systems, R. Tedrake and Y. Matsuoka, Eds., 2011, vol. 30, no. 1 , pp. 73-86.

[69] J. Fink, N. Michael, S. Kim, and V. Kumar, "Planning and control for cooperative manipulation and trasportation with aerial robots," The International Journal of Robotics Research, vol. 30, no. 3, pp. 324-334, 2011.

[70] K. Sreenath and V. Kumar, "Dynamics, control and planning for cooperative manipulation of payloads suspended by cables from multiple quadrotor robots," in Robotics: Science and Systems, Berlin, G, 2013.

[71] ARCAS, http://www.arcas-project.eu.

[72] R. Cano, C. Perez, F. Pruano, A. Oller, and G. Heredia, "Mechanical design of a 6-DOF aerial manipulator for assembling bar structures using UAVs," in 2nd RED-UAS Workshop on Research, Education and Development of Unmanned Aerial Systems, Compeigne, F, 2013.

[73] F. Ruggiero, M. Trujillo, R. Cano, H. Ascorbe, A. Viguria, C. Peréz, V. Lippiello, A. Ollero, and B. Siciliano, "A multilayer control for multirotor UAVs equipped with a servo robot arm," in 2015 IEEE 
International Conference on Robotics and Automation, Seattle, WA, USA, 2015, pp. 4014-4020.

[74] G. Heredia, A. Jimenez-Cano, I. Sanchez, D. Llorente, V. Vega, J. Braga, J. Acosta, and A. Ollero, "Control of a multirotor outdoor aerial manipulator," in 2014 IEEE/RSJ International Conference on Intelligent Robots and Systems, Chicago, IL, USA, 2014, pp. 34173422

[75] B. Yang, Y. He, J. Han, and G. Liu, "Rotor-flying manipulator: Modeling, analysis and control," Mathematical Problems in Engineering, vol. 1, no. 2, pp. 732-737, 2016.

[76] S. Kim, S. Choi, and H. Kim, "Aerial manipulation using a quadrotor with a two DOF robotic arm," in 2013 IEEE/RSJ International Conference on Intelligent Robots and Systems, Tokyo, J, 2013, pp. 4990-4995.

[77] F. Forte, R. Naldi, A. Macchelli, and L. Marconi, "Impedance control of an aerial manipulator," in American Control Conference, Montreal, C, 2012, pp. 3839-3844.

[78] A. Mersha, S. Stramigioli, and R. Carloni, "Exploiting the dynamics of a robotic manipulator for control of UAVs," in IEEE International Conference on Robotics and Automation, Hong Kong, C, 2014, pp. 1741-1746.

[79] A. Suarez, G. Heredia, and A. Ollero, "Lightweight compliant arm for aerial manipulation," in 2015 IEEE/RSJ International Conference on Intelligent Robots and Systems, Hamburg, D, 2015, pp. 1627-1632.

[80] C. Bellicoso, L. Buonocore, V. Lippiello, and B. Siciliano, "Design, modeling and control of a 5-DoF light-weight robot arm for aerial manipulation," in 23rd Mediterranean Conference on Control and Automation, Torremolinos, S, 2015, pp. 1741-1746.

[81] M. Fumagalli, R. Naldi, A. Macchelli, F. Forte, A. Keemink, S. Stramigioli, R. Carloni, and L. Marconi, "Developing an aerial manipulator prototype: Physical interaction with the environemnt," IEEE Robotics and Automation Magazine, vol. 21, no. 3, pp. 41-50, 2014.

[82] T. Danko, K. Chaney, and P. Oh, "A parallel manipulator for mobile manipulating UAVs," in 2015 IEEE International Conference on Technologies for Practical Robot Applications, Woburn, MA, USA, 2015.

[83] T. Danko and P. Oh, "A hyper-redundant manipulator for mobile manipulating unmanned aerial vehicles," in 2013 International Conference of Unmanned Aircraft Systems, Atlanta, GA, USA, 2013, pp. 974-981.

[84] F. Huber, K. Kondak, K. Krieger, D. Sommer, M. Schwarzbach, M. Laiacker, I. Kossyk, S. Parusel, S. Haddadin, and A. Albu-Schaffer, "First analysis and experiments in aerial manipulation using fully actuated redundant robot arm," in 2013 IEEE/RSJ International Conference on Intelligent Robots and Systems, Tokyo, J, 2013, pp. 3452-3457.

[85] Aeroarms, https://aeroarms-project.eu.

[86] A. Suarez, A. Jimenez-Cano, V. Vega, G. Heredia, A. RodriguezCastaño, and A. Ollero, "Lightweight and human-size dual arm aerial manipulator," in 2017 International Conference on Unmanned Aircraft Systems, Miami FL, USA, 2017, pp. 1778-1784.

[87] A. Suarez, P. Ramon, G. Heredia, B. Arrue, and A. Ollero, "Anthropomorphic, compliant and lightweight dual arm system for aerial manipulation," in 2017 IEEE/RSJ International Conference on Intelligent Robots and Systems, Vancouver, C, 2017.

[88] B. Yüksel, G. Buondonno, and A. Franchi, "Differential flatness and control of protocentric aerial manipulators with any number of arms and mixed rigid-/elastic joints," in 2016 IEEE/RSJ International Conference on Intelligent Robots and Systems, Daejeon, ROC, 2016, pp. 561-566.

[89] M. Orsag, C. Korpela, S. Bogdan, and P. Oh, "Valve turning using a dual-arm aerial manipulator," in 2014 International Conference on Unmanned Aircraft Systems, Orlando, FL, USA, 2014, pp. 836-841.

[90] C. Korpela, M. Orsag, and P. Oh, "Towards valve turning using a dualarm aerial manipulator," in 2014 IEEE/RSJ International Conference on Intelligent Robots and Systems, Chicago, IL, USA, 2014, pp. 34113416.

[91] PRODRONE, https://www.prodrone.jp/en/archives/1420/.

[92] V. Lippiello and F. Ruggiero, "Cartesian impedance control of a UAV with a robotic arm," in 10th International IFAC Symposium on Robot Control, Dubrovnik, HR, 2012, pp. 704-709.

[93] V. Lippiello and F. Ruggiero, "Exploiting redundancy in Cartesian impedance control of UAVs equipped with a robotic arm," in 2012 IEEE/RSJ International Conference on Intelligent Robots ans Systems, Vilamoura, P, 2012, pp. 3768-3773.

[94] S. Kannan, M. Olivares-Mendes, and H. Voos, "Modeling and control of aerial manipulation vehicle with visual sensor," in 2nd IFAC Workshop on Research, Education and Development of Unmanned Aerial Systems, Compeigne, F, 2013, pp. 303-309.
[95] G. Antonelli and E. Cataldi, "Adaptive control of arm-equipped quadrotors. Theory and simulations," in 2014 22nd Mediterranean Conference on Control and Automation, Palermo, I, 2014, pp. 1446-1451.

[96] M. Orsag, C. Korpela, S. Bogdan, and P. Oh, "Hybrid adaptive control for aerial manipulation," Journal of Intelligent \& Robotic Systems, vol. 73, no. 1-4, pp. 693-707, 2014.

[97] G. Arleo, F. Caccavale, G. Muscio, and F. Pierri, "Control of quadrotor aerial vehicles equipped witha robotic arm," in 21rd Mediterranean Conference on Control and Automation, Chania, GR, 2013, pp. 11741180.

[98] E. Cataldi, G. Muscio, M. Trujillo, Y. Rodriguez, F. Pierri, G. Antonelli, F. Caccavale, A. Viguria, S. Chiaverini, and A. Ollero, "Impedance control of an aerial-manipulator: Preliminary results," in 2016 IEEE/RSJ International Conference on Intelligent Robots and Systems, Daejeon, ROC, 2016, pp. 3848-3853.

[99] C. Korpela, M. Orsag, and P. Oh, "Hardware-in-the-loop verification for mobile manipulating unmanned aerial vehicles," Journal of Intelligent and Robotic Systems, vol. 73, no. 1-4, pp. 725-736, 2014

[100] C. Korpela, M. Orsag, and P. Oh, "Insertion tasks using an aerial manipulator," in 2014 IEEE International Conference on Technologies for Practical Robot Applications, Woburn, MA, USA, 2014, pp. 1-6.

[101] B. Siciliano, L. Sciavicco, L. Villani, and G. Oriolo, Robotics: Modelling, Planning and Control. London, UK: Springer, 2008.

[102] F. Ruggiero, J. Cacace, H. Sadeghian, and V. Lippiello, "Passivitybased control of VToL UAVs with a momentum-based estimator of external wrench and unmodeled dynamics," Robotics and Autonomous Systems, vol. 72, pp. 139-151, 2015.

[103] A. Jimenez-Cano, J. Martin, G. Heredia, A. Ollero, and R. Cano, "Control of an aerial robot with multi-link arm for assembly tasks," in IEEE International Conference on Robotics and Automation, Karlsruhe, D, 2013, pp. 4916-4921.

[104] E. Papadopoulos and S. Moosavian, "Dynamics and control of multiarm space robots during chase and capture operations," in 1994 IEEE/RSJ International Conference on Intelligent Robots and Systems, Munich, D, 1994, pp. 1554-1561.

[105] K. Yoshida, D. Dimitrov, and H. Nakanishi, "On the capture of tumbling satellite by a space robot," in 2006 IEEE/RSJ International Conference on Intelligent Robots and Systems, Beijing, C, 2006.

[106] F. Aghili, "Optimal control for robotic capturing and passivation of a tumbling satellite with unknown dynamics," in AIAA Guidance, Navigation and Control Conference and Exhibition, Honoluly, HI, USA, 2008.

[107] D. Reintsema, K. Landzettel, and G. Hirzinger, "DLR's advanced telerobotic concepts and experiments for on-orbit servicing," Advances in telerobotics, pp. 323-345, 2007.

[108] U. Hillenbrand and R. Lampariello, "Motion and parameter estimation of a free-floating space object from range data for motion prediction," in International Symposium on Artificial Intelligence, Robotics and Automation in Space, Munich, G, 2005.

[109] R. Lampariello, "Motion planning for the on-orbit grasping of a noncooperative target satellite with collision avoidance," in International Symposium on Artificial Intelligence, Robotics and Automation in Space, Sapporo, J, 2010, pp. 636-643.

[110] SPRINT Robotics, https://www.sprintrobotics.org/mediadownloads/sprint-robotics-strategic-roadmap.

[111] AIRobots, http://airobots.ing.unibo.it.

[112] SHERPA, http://www.sherpa-project.eu.

[113] EUROC, http://www.euroc-project.eu.

[114] Aeroworks, http://www.aeroworks2020.eu.

[115] 2017 Innovation Radar Prize winners, https://ec.europa.eu/digitalsingle-market/en/news/2017-innovation-radar-prize-winners. 